\title{
Dispersive estimates for Schrödinger operators in dimensions one and three
}

\author{
M. Goldberg, W. Schlag
}

\section{Introduction}

This paper deals with dispersive, i.e., $L^{1}\left(\mathbb{R}^{d}\right) \rightarrow L^{\infty}\left(\mathbb{R}^{d}\right)$ estimates for the time evolutions $e^{i t H} P_{a c}$ where $H=-\triangle+V$ and $P_{a c}$ is the projection onto the absolutely continuous spectral subspace. We restrict ourselves to the cases $d=1$ and $d=3$. Our goal is to assume as little as possible on the potential $V=V(x)$ in terms of decay or regularity. More precisely, we prove the following theorems.

Theorem 1. Let $V \in L_{1}^{1}(\mathbb{R})$, i.e., $\int_{-\infty}^{\infty}|V(x)|(1+|x|) d x<\infty$, and assume that there is no resonance at zero energy. Then for all $t$

$$
\left\|e^{i t H} P_{a c}(H)\right\|_{1 \rightarrow \infty} \lesssim|t|^{-\frac{1}{2}}
$$

where $H=-\frac{d^{2}}{d x^{2}}+V$. The conclusion holds for all $V \in L_{2}^{1}(\mathbb{R})$, i.e., $\int_{-\infty}^{\infty}|V(x)|(1+|x|)^{2} d x<\infty$, whether or not there is a resonance at zero energy.

A "resonance" here is defined to take place iff $W(0)=0$ where $W(\lambda)$ is the Wronskian of the two Jost solutions at energy $\lambda^{2}$, see the following section. It is known that the spectrum of $H$ is purely absolutely continuous on $(0, \infty)$ under our assumptions $\left(V \in L^{1}(\mathbb{R})\right.$ suffices for that) so that $P_{a c}$ is the same as the projection onto the orthogonal complement of the bound states. For the case of three dimensions we prove the following result.

Theorem 2. Let $|V(x)| \leq C(1+|x|)^{-\beta}$ for all $x \in \mathbb{R}^{3}$ where $\beta>3$. Assume also that zero is neither an eigenvalue nor a resonance of $H=-\triangle+V$. Then

$$
\left\|e^{i t H} P_{a c}(H)\right\|_{1 \rightarrow \infty} \lesssim|t|^{-\frac{3}{2}} .
$$

See Section 3 for a discussion of resonances. In this case, too, it is well-known that the spectrum is purely absolutely continuous on $[0, \infty)$.

Such dispersive estimates have a long history. For exponentially decaying potentials Rauch Rau proved dispersive bounds in exponentially weighted $L^{2}$-spaces. Jensen, Kato [JenKat] replaced exponential with polynomial decay and obtained asymptotic expansions of $e^{i t H}$ (in terms of powers of $t$ ) in the usual weighted $L^{2, \sigma}$ spaces. Journé, Soffer, and Sogge [JSS proved a version of Theorem 2 under the additional assumptions that $\beta>7, \hat{V} \in L^{1}$ and $V$ has some additional small amount of regularity. Yajima Yaj1 for the case $d=3$ proved that the wave operators are bounded on $L^{p}\left(\mathbb{R}^{3}\right)$ for all $1 \leq p \leq \infty$ assuming again that zero is neither an eigenvalue nor a resonance provided $\beta>5$ (and with similar conditions if $d>3$ ). As a consequence one obtains the $L^{1} \rightarrow L^{\infty}$ dispersive bounds. 
Our approach is very different from both [JSS] and Yaj1]. Journé, Soffer, and Sogge use a timedependent method and expand the evolution repeatedly by means of Duhamel's formula. For large energies the smallness needed to control the evolution $e^{i t H}$ appearing on the right-hand side of such an expansion is obtained from Kato's smoothing estimate. For small energies they use the expansion of the resolvent around zero energy from [JenKat. Since their method relies on the integrability of $t^{-\frac{d}{2}}$ at infinity, it can only be used in dimensions $d \geq 3$ and it also requires more regularity of $V$ $\left(\hat{V} \in L^{1}\right.$ is a natural assumption for their proof). Yajima Yaj1 uses the stationary approach of Kato [Kato to bound the wave operators on $L^{p}$. While his result is more general (it yields many more corollaries than just dispersive estimates), our approach to (2) is direct and also requires less of $V$. The one-dimensional case was open until recently. Weder Wed1 proved a version of Theorem 1 under the stronger assumption that $\int_{-\infty}^{\infty}|V(x)|(1+|x|)^{\frac{3}{2}+\varepsilon} d x<\infty$. Later, Weder Wed2, and also Artbazar, Yajima ArtYaj established corresponding theorems for the wave-operators. More precisely, they showed that the wave operators are bounded on $L^{p}(\mathbb{R})$ provided $1<p<\infty$ under similar assumptions on $V$. While our analysis is in some ways similar to Weder's Wed1, it turns out that the high energy case can be treated more easily by means of a Born series expansion, whereas small energies fall under the framework of the Jost solutions as developed in the fundamental paper by Deift, Trubowitz DeiTru]. The latter was also observed by Weder, but there is no need to impose any stronger condition on $V$ other than the one used in DeiTru, i.e., $V \in L_{1}^{1}(\mathbb{R})$.

Dispersive estimates in two dimensions are unknown in this degree of generality. Yajima Yaj2 established the $L^{p}\left(\mathbb{R}^{2}\right)$ boundedness of the wave operators under suitable assumptions on the decay of $V$ as well as the behavior of the Hamiltonian at zero energy. Since his result requires that $1<p<\infty$, it does not imply the $L^{1}\left(\mathbb{R}^{2}\right) \rightarrow L^{\infty}\left(\mathbb{R}^{2}\right)$ decay bounds for $e^{i t H} P_{a c}$, although it does imply the Strichartz estimates. We claim that our three-dimensional argument can be adapted to two dimensions as well, since it does not require integrability of $t^{-1}$ at infinity (unlike, say, [JSS]). Generally speaking, we expect the argument to apply to any dimension (in $d=1$, however, we use a different strategy which yields sharper results). For small energies we use expansions of the perturbed resolvent around zero energy. These were unknown in $\mathbb{R}^{2}$ for some time, but were recently obtained by Jensen, Nenciu [JenNen, whereas dimensions three and higher were treated by Jensen, Kato [JenKat] and Jensen [Jen1], Jen2]. We plan to present appropriate versions of Theorem 2 in dimensions two, or four and higher, elsewhere.

An interesting issue in Theorems 1 and 2 is the question of optimality. The decay rate of $(1+$ $|x|)^{-2-\varepsilon}$ appears to be a natural threshold for the dispersive estimates, and Theorem 1 achieves this rate. But we do not know at this point whether or not the statement of that theorem can also hold under weaker assumptions - the methods of proof used in this paper will certainly no longer apply for more slowly decaying potentials in the case of Theorem 11. On the other hand, it is possible that the methods employed in the proof of Theorem 2 do allow one to go below $\beta>3$. Let us remark that the weaker Strichartz estimates were shown to hold under the condition $\beta>2$ in RodSch by a completely different argument. 


\section{The one-dimensional case}

Let $H=-\frac{d^{2}}{d x^{2}}+V$ in $\mathbb{R}^{1}$. Our goal is to prove Theorem 1, It is well-known that for $V \in L^{1}(\mathbb{R}), H$ is essentially self-adjoint on the domain

$$
\left\{f \in L^{2}(\mathbb{R}) \mid f, f^{\prime} \text { are a.c. and }-f^{\prime \prime}+V f \in L^{2}(\mathbb{R})\right\}
$$

so that $e^{i t H}$ is unitary. Hence (10) is to be understood as the statement

$$
\left\|e^{i t H} P_{a c} f\right\|_{\infty} \lesssim|t|^{-\frac{1}{2}}\|f\|_{1} \text { for all } f \in L^{1}(\mathbb{R}) \cap L^{2}(\mathbb{R})
$$

which then extends to all of $L^{1}(\mathbb{R})$. We start with the high energy part of the argument.

Lemma 3. Let $\lambda_{0}=\|V\|_{1}^{2}$ and suppose $\chi$ is a smooth cut-off such that $\chi(\lambda)=0$ for $\lambda \leq \lambda_{0}$ and $\chi(\lambda)=1$ for $\lambda \geq 2 \lambda_{0}$. Then

$$
\left\|e^{i t H} \chi(H)\right\|_{1 \rightarrow \infty} \lesssim|t|^{-\frac{1}{2}}
$$

for all $t$.

Proof. In the limit $\varepsilon \rightarrow 0+$ the one-dimensional resolvent $R_{0}(\lambda+i \varepsilon):=\left(-\frac{d^{2}}{d x^{2}}-(\lambda+i \varepsilon)\right)^{-1}$ has the kernel

$$
R_{0}(\lambda \pm i 0)(x)=\frac{ \pm i}{2 \sqrt{\lambda}} e^{ \pm i|x| \sqrt{\lambda}}
$$

Because of the decay of this kernel in $\lambda$, the resolvent $R_{V}(\lambda+i \varepsilon)=(H-(\lambda+i \varepsilon))^{-1}$ can be expanded into the Born series

$$
R_{V}(\lambda \pm i 0)=\sum_{n=0}^{\infty} R_{0}(\lambda \pm i 0)\left(-V R_{0}(\lambda \pm i 0)\right)^{n} .
$$

More precisely, since $\left\|V R_{0}(\lambda \pm i 0)\right\|_{1 \rightarrow 1} \leq(2 \sqrt{\lambda})^{-1}\|V\|_{1}$, one has

$$
\left|\left\langle R_{0}(\lambda+i 0)\left(V R_{0}(\lambda+i 0)\right)^{n} f, g\right\rangle\right| \leq(2 \sqrt{\lambda})^{-n-1}\|V\|_{1}^{n}\|f\|_{1}\|g\|_{1},
$$

so that (41) converges provided $\lambda>\lambda_{0}=\|V\|_{1}^{2}$ in the following weak sense:

$$
\left\langle R_{V}(\lambda \pm i 0) f, g\right\rangle=\sum_{n=0}^{\infty}\left\langle R_{0}(\lambda \pm i 0)\left(-V R_{0}(\lambda \pm i 0)\right)^{n} f, g\right\rangle
$$

for any pair of $L^{1}$ functions $f, g$. For such functions it is a standard fact that

$$
R_{V}(\lambda-i 0) g \in L^{\infty}(\mathbb{R})
$$

provided $\lambda>0$ (this follows, for example, from the boundedness of the Jost solutions, see below). Therefore, the error term in any finite Born expansion, i.e., $R_{V}(\lambda+i 0)\left(V R_{0}(\lambda+i 0)\right)^{n}$, tends to zero weakly as $n \rightarrow \infty$ provided $\lambda>\lambda_{0}$ since

$$
\begin{aligned}
& \left|\left\langle R_{V}(\lambda+i 0)\left(V R_{0}(\lambda+i 0)\right)^{n} f, g\right\rangle\right| \leq\left\|\left(V R_{0}(\lambda+i 0)\right)^{n} f\right\|_{1}\left\|R_{V}(\lambda-i 0) g\right\|_{\infty} \\
& \leq(2 \sqrt{\lambda})^{-n}\|V\|_{1}^{n}\|f\|_{1}\left\|R_{V}(\lambda-i 0) g\right\|_{\infty} .
\end{aligned}
$$


For technical reasons we introduce a truncated version $\chi_{L}$ of the cut-off $\chi: \chi_{L}(\lambda)=\chi(\lambda) \phi(\lambda / L)$ where $\phi$ is smooth, $\phi(\lambda)=1$ if $|\lambda| \leq 1, \phi(\lambda)=0$ if $|\lambda| \geq 2$, and $L \geq 1$. We need to show that

$$
\sup _{L \geq 1}\left|\left\langle e^{i t H} \chi_{L}(H) f, g\right\rangle\right| \leq C|t|^{-\frac{1}{2}}\|f\|_{1}\|g\|_{1}
$$

for any pair of Schwartz functions $f, g$. The absolutely continuous part of the spectral measure of $H$, which we denote by $E_{a c}(d \lambda)$, and the resolvent $R_{V}(\lambda+i 0)$ are related by the well-known formula

$$
\left\langle E_{a c}(d \lambda) f, g\right\rangle=\left\langle\frac{1}{2 \pi i}\left[R_{V}(\lambda+i 0)-R_{V}(\lambda-i 0)\right] f, g\right\rangle d \lambda .
$$

Since $\chi_{L}(H) E(d \lambda)=\chi_{L}(H) E_{a c}(d \lambda)$ one concludes that

$$
\left|\left\langle e^{i t H} \chi_{L}(H) f, g\right\rangle\right|=\left|(2 \pi i)^{-1} \sum_{n=0}^{\infty} \int_{-\infty}^{\infty} e^{i t \lambda^{2}} \chi_{L}\left(\lambda^{2}\right) \lambda\left\langle R_{0}\left(\lambda^{2}+i 0\right)\left(V R_{0}\left(\lambda^{2}+i 0\right)\right)^{n} f, g\right\rangle d \lambda\right|
$$

where we have first changed variables $\lambda \rightarrow \lambda^{2}$. Summation and integration may be exchanged because the Born series converges absolutely in the $L_{\text {loc }}^{1}(d \lambda)$ norm, and the domain of integration is extended to $\mathbb{R}$ from $[0, \infty)$ via the identity $R_{0}\left(\lambda^{2}-i 0\right)=R_{0}\left((-\lambda)^{2}+i 0\right)$ (where $R_{0}\left(z^{2}\right)$ is interpreted as an analytic function for $z \neq 0$, see (3) $)$. The kernel of $R_{0}\left(\lambda^{2}+i 0\right)\left(V R_{0}\left(\lambda^{2}+i 0\right)\right)^{n}$ is given explicitly by the formula

$$
R_{0}\left(\lambda^{2}+i 0\right)\left(V R_{0}\left(\lambda^{2}+i 0\right)\right)^{n}(x, y)=\frac{1}{(2 \lambda)^{n+1}} \int_{\mathbb{R}^{n}} \prod_{j=1}^{n} V\left(x_{j}\right) e^{i \lambda\left(\left|x-x_{1}\right|+\left|y-x_{n}\right|+\sum_{k=2}^{n}\left|x_{k}-x_{k-1}\right|\right)} d x_{1} \ldots d x_{n}
$$

Fubini's theorem permits integration in $d \lambda$ prior to all of the $d x_{j}$, leading to the desired bound

$$
\begin{aligned}
\left|\left\langle e^{i t H} \chi_{L}(H) f, g\right\rangle\right| & \lesssim \sum_{n=0}^{\infty}\left(2 \sqrt{\lambda_{0}}\right)^{-n} \sup _{a \in \mathbb{R}}\left|\int_{-\infty}^{\infty} e^{i\left(t \lambda^{2}+a \lambda\right)} \chi_{L}\left(\lambda^{2}\right) \lambda^{-n} \lambda_{0}^{n / 2} d \lambda\right|\|V\|_{1}^{n}\|f\|_{1}\|g\|_{1} \\
& \leq C\left(V_{1}\right)|t|^{-\frac{1}{2}}\|f\|_{1}\|g\|_{1} .
\end{aligned}
$$

We have used the dispersive bound for the one-dimensional Schrödinger equation to estimate the oscillatory integral in (8). Indeed, the quantity inside the absolute values is the solution of a onedimensional Schrödinger equation at time $t$ and position $a$ with initial data $\left[\chi_{L}\left(\lambda^{2}\right) \lambda^{-n} \lambda_{0}^{(n+1) / 2}\right]^{\vee}$. In order to pass to (9), it therefore remains to check that

$$
\sup _{n \geq 0} \sup _{L \geq 1}\left\|\left[\chi_{L}\left(\lambda^{2}\right) \lambda^{-n} \lambda_{0}^{n / 2}\right]^{\vee}\right\|_{1}<\infty
$$

For $n=0$ this reduces to

$$
\left\|\left[\chi_{L}\left(\lambda^{2}\right)\right]^{\vee}\right\|_{1} \leq\left\|\widehat{L\left(\lambda^{2}\right)}(L \xi)\right\|_{1}\left(1+\left\|\left(1-\chi\left(\lambda^{2}\right)\right)^{\vee}\right\|_{1}\right)<\infty
$$

uniformly in $L$ since $1-\chi$ is compactly supported and smooth. For general $n$ one has

$$
\left\|\left[\chi_{L}\left(\lambda^{2}\right) \lambda^{-n}\right]^{\vee}(\tau) \tau^{2}\right\|_{\infty}=\left\|\left[\left(\chi_{L}\left(\lambda^{2}\right) \lambda^{-n}\right)^{\prime \prime}\right]^{\vee}(\tau)\right\|_{\infty} \leq\left\|\left(\chi_{L}\left(\lambda^{2}\right) \lambda^{-n}\right)^{\prime \prime}\right\|_{1} \leq C\left(\lambda_{0}\right) \lambda_{0}^{-n / 2},
$$


where the constant $C_{0}(\lambda)$ only depends on $\lambda_{0}$, but not on $n$ or $L$. For $n \geq 2$ one also has

$$
\left\|\left[\chi_{L}\left(\lambda^{2}\right) \lambda^{-n}\right]^{\vee}(\tau)\right\|_{\infty} \leq\left\|\chi_{L}\left(\lambda^{2}\right) \lambda^{-n}\right\|_{1} \leq C\left(\lambda_{0}\right) \lambda_{0}^{-n / 2}
$$

so that (10) follows for $n \geq 2$. It remains to check that $\left\|\left[\chi_{L}\left(\lambda^{2}\right) \lambda^{-1}\right]^{\vee}(\tau)\right\|_{\infty}<\infty$ uniformly in $L$. However,

$$
\left\|\left[\chi_{L}\left(\lambda^{2}\right) \lambda^{-1}\right]^{\vee}\right\|_{\infty} \leq\left\|\left[\chi_{L}\left(\lambda^{2}\right)\right]^{\vee}\right\|_{1}\left\|\left[\lambda^{-1}\right]^{\vee}\right\|_{\infty}<\infty
$$

uniformly in $L \geq 1$ by (11) and $\left[\lambda^{-1}\right]^{\vee}(\xi)=-i \operatorname{sign}(\xi)$.

For the low energy part we use the Jost solutions $f_{ \pm}(z, \cdot)$. They are defined as solutions of

$$
-f_{ \pm}^{\prime \prime}(z, x)+V(x) f_{ \pm}(z, x)=z^{2} f_{ \pm}(z, x)
$$

for $\Im z \geq 0$ satisfying $\left|f_{ \pm}(z, x)-e^{ \pm i z x}\right| \rightarrow 0$ as $x \rightarrow \pm \infty$. In what follows we set $z=\lambda \in \mathbb{R}$. They are known to exist for $\lambda \neq 0$ if $V \in L^{1}(\mathbb{R})$. If $V \in L_{1}^{1}$, then they also exist at $\lambda=0$. Denote their Wronskian by $W(\lambda)=W\left[f_{+}(\lambda, \cdot), f_{-}(\lambda, \cdot)\right]$. It is well-known DeiTru that $W(\lambda) \neq 0$ if $\lambda \neq 0$. The Green's function has kernel

$$
\left(H-\left(\lambda^{2} \pm i 0\right)\right)^{-1}(x, y)=R_{V}\left(\lambda^{2} \pm i 0\right)(x, y)=\frac{f_{+}( \pm \lambda, y) f_{-}( \pm \lambda, x)}{W( \pm \lambda)}
$$

for all $\lambda \neq 0$ and $x<y$ (and the positions of $x, y$ reversed if $x>y$ ). If $W(0)=0$ we say that zero energy is a resonance. Therefore, in the non-resonant case, for any $x<y$, and any smooth, compactly supported (say) cut-off $\chi$

$$
\begin{aligned}
& 2 \pi i \int_{0}^{\infty} e^{i t \lambda} \chi(\lambda) E_{a . c .}(d \lambda)(x, y)=\int_{0}^{\infty} e^{i t \lambda^{2}} \lambda \chi\left(\lambda^{2}\right)\left[\frac{f_{+}(\lambda, y) f_{-}(\lambda, x)}{W(\lambda)}-\frac{f_{+}(-\lambda, y) f_{-}(-\lambda, x)}{W(-\lambda)}\right] d \lambda \\
& =\int_{-\infty}^{\infty} e^{i t \lambda^{2}} \lambda \chi\left(\lambda^{2}\right) \frac{f_{+}(\lambda, y) f_{-}(\lambda, x)}{W(\lambda)} d \lambda .
\end{aligned}
$$

In view of Lemma 3 the non-resonant part of Theorem 1 will follow from the following low-energy lemma.

Lemma 4. Let $V \in L_{1}^{1}(\mathbb{R})$ and $W(0) \neq 0$. Then

$$
\sup _{x<y}\left|\int_{-\infty}^{\infty} e^{i t \lambda^{2}} \frac{\lambda \chi(\lambda)}{W(\lambda)} f_{+}(\lambda, y) f_{-}(\lambda, x) d \lambda\right| \lesssim|t|^{-\frac{1}{2}}
$$

for any $t$. Here $\chi$ is any smooth, compactly supported cut-off.

We will distinguish the cases $x<0<y, 0<x<y$, and $x<y<0$. Write

$$
f_{ \pm}(\lambda, x)=e^{ \pm i \lambda x} m_{ \pm}(\lambda, x)
$$

so that $\left|m_{ \pm}(\lambda, x)-1\right| \rightarrow 0$ as $x \rightarrow \pm \infty$. It is known, see DeiTru, that $m_{ \pm}(z, x)-1$ as a function of $z$ belongs to $H^{2}\left(\mathbb{C}^{+}\right)$, the Hardy space on the upper half plane. Moreover, $m_{ \pm}(\hat{\xi}, x)-\delta_{0}(\xi) \in \mathcal{M}$ relative to $\xi$ where $m_{ \pm}(\hat{\xi}, x)$ denotes the Fourier transform in the first variable alone and $\mathcal{M}$ are the 
(complex) measures. By the $H^{2}$ property, $m_{ \pm}(\hat{\xi}, x)$ is supported in $\xi \geq 0$. A number of pointwise estimates can be made for $m_{ \pm}(\hat{\xi}, x)$. Define $I(\xi):=\int_{|t|>|\xi|}|V(t)| d t$. Then

$$
\begin{array}{clrl}
\sup _{x \geq 0}\left|m_{+}(\hat{\xi}, x)-\delta_{0}\right| \lesssim I(\xi), & & \sup _{x \leq 0}\left|m_{-}(\hat{\xi}, x)-\delta_{0}\right| \lesssim I(\xi), \\
x \geq 0 \Rightarrow\left|\partial_{x} m_{+}(\hat{\xi}, x)\right| \lesssim I(\xi)+|V(x+\xi)|, & & \left|\partial_{\xi}\left(m_{+}(\hat{\xi}, x)-\delta_{0}\right)\right| \lesssim I(\xi)+|V(x+\xi)|, \\
x \leq 0 \Rightarrow\left|\partial_{x} m_{-}(\hat{\xi}, x)\right| \lesssim I(\xi)+|V(x-\xi)|, & & \left|\partial_{\xi}\left(m_{-}(\hat{\xi}, x)-\delta_{0}\right)\right| \lesssim I(\xi)+|V(x-\xi)|,
\end{array}
$$

see Lemma 3 in DeiTru. The assumption $V \in L_{1}^{1}(\mathbb{R})$ suffices to bound the total variation norms $\left\|m_{+}(\hat{\imath}, x)\right\|_{\mathcal{M}},\left\|\partial_{x} m_{+}(\hat{\imath}, x)\right\|_{\mathcal{M}}$, and $\left\|\partial_{\xi}\left(m_{+}(\hat{\imath}, x)-\delta_{0}\right)\right\|_{\mathcal{M}}$ uniformly in $x \geq 0$. Similarly, the norms $\left\|m_{-}(\hat{\imath}, x)\right\|_{\mathcal{M}},\left\|\partial_{x} m_{-}(\hat{\imath}, x)\right\|_{\mathcal{M}}$, and $\left\|\partial_{\xi}\left(m_{-}(\hat{\imath}, x)-\delta_{0}\right)\right\|_{\mathcal{M}}$ are bounded uniformly in $x \leq 0$. Identical bounds are also true of $\left[\chi(\cdot) m_{ \pm}(\cdot, x)\right]^{\wedge}(\xi)$, however the convolution with $\hat{\chi}$ provides enough smoothing that the norms may be taken in $L^{1}(\xi)$, and the point-mass correction $\delta_{0}$ is not needed.

If $V$ satisfies the stronger hypothesis $V \in L_{2}^{1}(\mathbb{R})$, then $I \in L_{1}^{1}(\mathbb{R})$ leading to uniform bounds for $m_{ \pm}(\hat{\xi}, x)$ and its derivatives in the $L_{1}^{1}(\mathbb{R})$ norm. Note, however, that these suprema are typically not finite if they are taken over all $x \in \mathbb{R}$ rather than on the appropriate half-line.

Lemma 5. Let $V \in L_{j}^{1}(\mathbb{R}), j=1,2$, and $\tilde{\chi}$ a smooth, compactly supported cut-off which is identically 1 on the support of $\chi$. Then the functions $\tilde{\chi}(\lambda) W(\lambda)$ and $W\left[f_{+}(\lambda, \cdot), f_{-}(-\lambda, \cdot)\right]$ both have Fourier transform in $L_{(j-1)}^{1}(\mathbb{R})$.

Proof. By definition,

$$
\begin{aligned}
& \tilde{\chi}(\lambda) W(\lambda)=\tilde{\chi}(\lambda)\left(m_{+}(\lambda, 0) \partial_{x} m_{-}(\lambda, 0)-\partial_{x} m_{+}(\lambda, 0) m_{-}(\lambda, 0)\right)-2 i \lambda \tilde{\chi}(\lambda) m_{+}(\lambda, 0) m_{-}(\lambda, 0) \\
& W\left[f_{+}(\lambda, \cdot), f_{-}(-\lambda, \cdot)\right]=m_{+}(\lambda, 0) \partial_{x} m_{-}(-\lambda, 0)-\partial_{x} m_{+}(\lambda, 0) m_{-}(-\lambda, 0)
\end{aligned}
$$

The estimates in (13) suffice to prove the lemma, since the Fourier transform of each product will be a convolution of functions in $L_{(j-1)}^{1}(\mathbb{R})$.

Proof of Lemma 4. In the case $x<0<y$,

$$
\begin{aligned}
& \sup _{x<0<y}\left|\int_{-\infty}^{\infty} e^{i t \lambda^{2}} \frac{\lambda \chi(\lambda)}{W(\lambda)} f_{+}(\lambda, y) f_{-}(\lambda, x) d \lambda\right| \\
& =\sup _{x<0<y}\left|\int_{-\infty}^{\infty} e^{i t \lambda^{2}} e^{i \lambda(x-y)} \frac{\lambda \chi(\lambda)}{\tilde{\chi}(\lambda) W(\lambda)} m_{+}(\lambda, y) m_{-}(\lambda, x) d \lambda\right| \lesssim|t|^{-\frac{1}{2}} .
\end{aligned}
$$

The final inequality follows again from the dispersive bound for the one-dimensional Schrödinger equation provided

$$
\sup _{x<0<y}\left\|\left[\frac{\lambda \chi(\lambda)}{\tilde{\chi}(\lambda) W(\lambda)} m_{+}(\lambda, y) m_{-}(\lambda, x)\right]^{\vee}\right\|<\infty
$$

where the Fourier transform is with respect to $\lambda$ alone, and the norm is in the sense of measures. Since $(\tilde{\chi} W)^{\vee} \in L^{1}(\mathbb{R})$ by Lemma 5. Wiener's lemma (see Katz chapter VIII Lemma 6.3) implies that $\frac{\lambda \chi(\lambda)}{\tilde{\chi}(\lambda) W(\lambda)}$ is the Fourier transform of an $L^{1}(\mathbb{R})$ function, and therefore (15) holds. 
By symmetry, it suffices to check the remaining case $0 \leq x<y$. The danger here is that $f_{-}(0, x)$ can grow as $x \rightarrow \infty$. To deal with this issue, we expand

$$
f_{-}(\lambda, x)=\alpha_{-}(\lambda) f_{+}(\lambda, x)+\beta(\lambda) f_{+}(-\lambda, x)
$$

where $\beta(\lambda)=\frac{W(\lambda)}{-2 i \lambda}$ and $\alpha_{-}(\lambda)=\frac{-1}{2 i \lambda} W\left[f_{-}(\lambda, \cdot), f_{+}(-\lambda, \cdot)\right]$. Hence

$$
\begin{aligned}
& \sup _{0 \leq x<y}\left|\int_{-\infty}^{\infty} e^{i t \lambda^{2}} \frac{\lambda \chi(\lambda)}{\tilde{\chi}(\lambda) W(\lambda)} f_{+}(\lambda, y) f_{-}(\lambda, x) d \lambda\right| \\
& \lesssim \sup _{0 \leq x<y}\left|\int_{-\infty}^{\infty} e^{i t \lambda^{2}} e^{i \lambda(x+y)} \frac{\lambda \alpha_{-}(\lambda)}{\tilde{\chi}(\lambda) W(\lambda)} \chi(\lambda) m_{+}(\lambda, y) m_{+}(\lambda, x) d \lambda\right| \\
& +\sup _{0 \leq x<y}\left|\int_{-\infty}^{\infty} e^{i t \lambda^{2}} e^{i \lambda(y-x)} \chi(\lambda) m_{+}(\lambda, y) m_{+}(-\lambda, x) d \lambda\right| \lesssim|t|^{-\frac{1}{2}}
\end{aligned}
$$

where the final inequality again follows by noting that $\lambda \alpha_{-}(\lambda)$ has Fourier transform in $L^{1}(\mathbb{R})$ and invoking the Wiener algebra.

In the resonant case of Theorem 11. $W(0)$ vanishes and we cannot apply the Wiener lemma to expressions with $W(\lambda)$ in the denominator. The additional hypothesis $V \in L_{2}^{1}(\mathbb{R})$ ensures that $\frac{W(\lambda)}{\lambda}$ is continuous and nonzero everywhere (see Theorem 1 in DeiTru]). The Fourier transform of $\tilde{\chi}(\lambda) W(\lambda)$ is in $L_{1}^{1}(\mathbb{R})$ by Lemma $[5$ and furthermore by the identity

$$
\left(\frac{\tilde{\chi} W}{\lambda}\right)^{\vee}(\xi)=i\left[\int_{\xi}^{\infty}(\tilde{\chi} W)^{\vee}(\eta) d \eta-\int_{-\infty}^{\xi}(\tilde{\chi} W)^{\vee}(\eta) d \eta\right]
$$

it follows that $(\tilde{\chi} \beta)^{\vee} \in L^{1}(\mathbb{R})$ (keeping in mind that $\int_{-\infty}^{\infty}(\tilde{\chi} W)^{\vee}(\eta) d \eta=0$ ). A similar argument shows that $\left(\alpha_{-}\right)^{\vee} \in L^{1}(\mathbb{R})$. By rewriting every fraction with denominator $\tilde{\chi}(\lambda) W(\lambda)$ to have instead a denominator of $\tilde{\chi}(\lambda) \beta(\lambda)$ (this is done by canceling a common factor of $\lambda$ in the numerator and denominator), the Wiener lemma may be applied precisely as above.

\section{The three-dimensional case}

Let $H=-\Delta+V$ in $\mathbb{R}^{3}$. Our goal is to prove Theorem 2, We first recall the definition of a resonance. The meaning of this notion will become clear later.

Definition 6. As usual, we say that a resonance occurs at zero, provided there is a distributional solution $f$ of the equation $(-\triangle+V) f=0$ where for every $\sigma<-\frac{1}{2}$ one has $f \in L^{2, \sigma}\left(\mathbb{R}^{3}\right) \backslash L^{2}\left(\mathbb{R}^{3}\right)$.

Let $\chi$ be a smooth, even, cut-off function on the line that is equal to one on a neighborhood of the origin. Then, with $R_{0}(z):=(-\Delta-z)^{-1}$ and $R_{V}(z):=(H-z)^{-1}$, we need to prove that

$$
\begin{aligned}
\sup _{L \geq 1}\left|\left\langle e^{i t H} \chi(\sqrt{H} / L) P_{a . c .} f, g\right\rangle\right| & =\sup _{L \geq 1}\left|\int_{0}^{\infty} e^{i t \lambda^{2}} \lambda \chi(\lambda / L)\left\langle\left[R_{V}\left(\lambda^{2}+i 0\right)-R_{V}\left(\lambda^{2}-i 0\right)\right] f, g\right\rangle \frac{d \lambda}{\pi i}\right| \\
& \lesssim|t|^{-\frac{3}{2}}\|f\|_{1}\|g\|_{1},
\end{aligned}
$$


see (17). Iterating the resolvent identity yields the finite Born series

$$
\begin{aligned}
R_{V}\left(\lambda^{2} \pm i 0\right) & =\sum_{\ell=0}^{2 m+1} R_{0}\left(\lambda^{2} \pm i 0\right)\left(-V R_{0}\left(\lambda^{2} \pm i 0\right)\right)^{\ell} \\
& +R_{0}\left(\lambda^{2} \pm i 0\right)\left(V R_{0}\left(\lambda^{2} \pm i 0\right)\right)^{m} V R_{V}\left(\lambda^{2} \pm i 0\right) V\left(R_{0}\left(\lambda^{2} \pm i 0\right) V\right)^{m} R_{0}\left(\lambda^{2} \pm i 0\right) .
\end{aligned}
$$

Here $m$ is any positive integer. One needs to distinguish small $0<\lambda<\lambda_{0}$ from $\lambda>\lambda_{0}$, where $\lambda_{0}>0$ is a small constant that will be determined by the small energy considerations below. In the latter case, use the limiting absorption principle. In the former case, one expands the resolvent $R_{V}$ around zero energy as in Jensen, Kato JenKat. This requires assuming that zero is neither an eigenvalue nor a resonance. We will, however, not rely on JenKat but rederive the expansion of the resolvent ourselves in the form needed here.

\subsection{Large energies}

We now turn to the large energy estimates which will yield the desired bound on

$$
\begin{aligned}
& \left\langle e^{i t H} \chi\left(\frac{\sqrt{H}}{L}\right)\left[1-\chi\left(\frac{\sqrt{H}}{\lambda_{0}}\right)\right] P_{a . c .} f, g\right\rangle \\
& =\int_{0}^{\infty} e^{i t \lambda^{2}} \lambda \chi\left(\frac{\lambda}{L}\right)\left[1-\chi\left(\frac{\lambda}{\lambda_{0}}\right)\right]\left\langle\left[R_{V}\left(\lambda^{2}+i 0\right)-R_{V}\left(\lambda^{2}-i 0\right)\right] f, g\right\rangle \frac{d \lambda}{\pi i},
\end{aligned}
$$

cf. (77). Insert the resolvent expansion (17) into (18). The first $2 m+2$ terms which do not contain the resolvent $R_{V}$ are treated as in RodSch], Section 2. This only requires that

$$
\|V\|_{\mathcal{K}}:=\sup _{x \in \mathbb{R}^{3}} \int \frac{|V(y)|}{|x-y|} d y<\infty
$$

In particular, if $|V(x)| \lesssim(1+|x|)^{-2-\varepsilon}$, then this condition is satisfied. The method from RodSch gives an $L^{1}\left(\mathbb{R}^{3}\right) \rightarrow L^{\infty}\left(\mathbb{R}^{3}\right)$ bound with decay $|t|^{-\frac{3}{2}}$ for those terms. For the convenience of the reader

we recall the relevant parts from $[$ RodSch. The contribution by the $(k+1)$-st term in the Born series (17) is equal to

$$
\begin{aligned}
& \int_{0}^{\infty} e^{i t \lambda} \psi(\sqrt{\lambda} / L)\left(1-\chi\left(\sqrt{\lambda} / \lambda_{0}\right)\right)\left\langle R_{0}(\lambda+i 0)\left(V R_{0}(\lambda+i 0)\right)^{k} f, g\right\rangle d \lambda \\
& -\int_{0}^{\infty} e^{i t \lambda} \psi(\sqrt{\lambda} / L)\left(1-\chi\left(\sqrt{\lambda} / \lambda_{0}\right)\right)\left\langle R_{0}(\lambda-i 0)\left(V R_{0}(\lambda-i 0)\right)^{k} f, g\right\rangle d \lambda
\end{aligned}
$$


which is controlled by

$$
\begin{aligned}
& \sup _{L \geq 1}\left|\int_{0}^{\infty} e^{i t \lambda} \psi(\sqrt{\lambda} / L)\left(1-\chi\left(\sqrt{\lambda} / \lambda_{0}\right)\right) \Im\left\langle R_{0}(\lambda+i 0)\left(V R_{0}(\lambda+i 0)\right)^{k} f, g\right\rangle d \lambda\right| \\
& \leq \int_{\mathbb{R}^{6}}\left|f\left(x_{0}\right)\right|\left|g\left(x_{k+1}\right)\right| \int_{\mathbb{R}^{3 k}} \frac{\prod_{j=1}^{k}\left|V\left(x_{j}\right)\right|}{\prod_{j=0}^{k} 4 \pi\left|x_{j}-x_{j+1}\right|} .
\end{aligned}
$$

$$
\leq C t^{-\frac{3}{2}} \int_{\mathbb{R}^{6}}\left|f\left(x_{0}\right)\right|\left|g\left(x_{k+1}\right)\right| \int_{\mathbb{R}^{3 k}} \frac{\prod_{j=1}^{k}\left|V\left(x_{j}\right)\right|}{(4 \pi)^{k+1} \prod_{j=0}^{k}\left|x_{j}-x_{j+1}\right|} \sum_{\ell=0}^{k}\left|x_{\ell}-x_{\ell+1}\right| d\left(x_{1}, \ldots, x_{k}\right) d x_{0} d x_{k+1}
$$

$$
\begin{aligned}
& \leq C t^{-\frac{3}{2}} \int_{\mathbb{R}^{6}}\left|f\left(x_{0}\right) \| g\left(x_{k+1}\right)\right|(k+1)\left(\|V\|_{\mathcal{K}} / 4 \pi\right)^{k} d x_{0} d x_{k+1} \\
& \leq C_{k} t^{-\frac{3}{2}}\|f\|_{1}\|g\|_{1} .
\end{aligned}
$$

In order to pass to (20) one uses the explicit representation of the kernel of $R_{0}(\lambda+i 0)(x, y)=\frac{e^{i \lambda|\mathbf{x}-\mathbf{y}|}}{4 \pi|\mathbf{x}-\mathbf{y}|}$, which leads to a $k$-fold integral. The inequalities (21) and (22) are obtained by means of the following two lemmas from RodSch]. We provide the proof of the first lemma, as its statement differs slightly from the one in RodSch (by the introduction of an additional zero energy cut-off).

Lemma 7. Let $\psi$ be a smooth, even bump function with $\psi(\lambda)=1$ for $-1 \leq \lambda \leq 1$ and $\operatorname{supp}(\psi) \subset$ $[-2,2]$. Then for all $t \geq 1$ and any real $a$,

$$
\sup _{L \geq 1}\left|\int_{0}^{\infty} e^{i t \lambda} \sin (a \sqrt{\lambda}) \psi\left(\frac{\sqrt{\lambda}}{L}\right)\left(1-\chi\left(\sqrt{\lambda} / \lambda_{0}\right)\right) d \lambda\right| \leq C t^{-\frac{3}{2}}|a|
$$

where $C$ only depends on $\psi, \chi$, and $\lambda_{0}$.

Proof. Denote the integral in (23) by $I_{L}(a, t)$. The change of variables $\lambda \rightarrow \lambda^{2}$ leads to the expression

$$
I_{L}(a, t)=2 \int_{0}^{\infty} \lambda e^{i t \lambda^{2}} \sin (a \lambda) \psi(\lambda / L)\left(1-\chi\left(\lambda / \lambda_{0}\right)\right) d \lambda
$$

Integrating by parts we obtain

$$
I_{L}(a, t)=-\frac{i}{t} \int_{0}^{\infty} e^{i t \lambda^{2}}\left(a \cos (a \lambda) \psi(\lambda / L)\left(1-\chi\left(\lambda / \lambda_{0}\right)\right)+\sin (a \lambda)\left[\left(1-\chi\left(\lambda / \lambda_{0}\right)\right) \psi(\lambda / L)\right]^{\prime}\right) d \lambda .
$$

Since $\psi$ and $\chi$ are assumed to be even, the derivative of the brackets is odd. Hence,

$$
\begin{aligned}
I_{L}(a, t)= & -\frac{i}{2 t} \int_{-\infty}^{\infty} e^{i t \lambda^{2}}\left(a \cos (a \lambda)\left(1-\chi\left(\lambda / \lambda_{0}\right)\right) \psi(\lambda / L)+\sin (a \lambda)\left[\left(1-\chi\left(\lambda / \lambda_{0}\right)\right) \psi(\lambda / L)\right]^{\prime}\right) d \lambda \\
= & -\frac{a}{4 t} i \int_{-\infty}^{\infty} e^{i t \lambda^{2}}\left(e^{i a \lambda}+e^{-i a \lambda}\right) \psi(\lambda / L)\left(1-\chi\left(\lambda / \lambda_{0}\right)\right) d \lambda \\
& +\int_{0}^{a} \frac{1}{4 t} \int_{-\infty}^{\infty} e^{i t \lambda^{2}}\left(e^{i b \lambda}+e^{-i b \lambda}\right) \lambda\left[\left(1-\chi\left(\lambda / \lambda_{0}\right)\right) \psi(\lambda / L)\right]^{\prime} d \lambda d b .
\end{aligned}
$$


Invoking the $|t|^{-\frac{1}{2}}$ dispersive bound for the one-dimensional Schrödinger equation, it thus suffices to show that

$$
\left\|\left[\left(1-\chi\left(\lambda / \lambda_{0}\right)\right) \psi(\lambda / L)\right]^{\vee}\right\|_{1}+\left\|\left[\lambda\left[\left(1-\chi\left(\lambda / \lambda_{0}\right)\right) \psi(\lambda / L)\right]^{\prime}\right]^{\vee}\right\|_{1}<\infty
$$

uniformly in $L \geq 1$. These properties are elementary and left to the reader.

The following lemma is identical with one in Section 2 of [RodSch], and we refer the reader to that paper for the simple proof.

Lemma 8. For any positive integer $k$ and $V$ as in (19)

$$
\sup _{x_{0}, x_{k+1} \in \mathbb{R}^{3}} \int_{\mathbb{R}^{3 k}} \frac{\prod_{j=1}^{k}\left|V\left(x_{j}\right)\right|}{\prod_{j=0}^{k}\left|x_{j}-x_{j+1}\right|} \sum_{\ell=0}^{k}\left|x_{\ell}-x_{\ell+1}\right| d x_{1} \ldots d x_{k} \leq(k+1)\|V\|_{\mathcal{K}}^{k} .
$$

We now turn to the term in the Born series (17) containing the perturbed resolvent $R_{V}$. Recall from Agmon $\mathrm{Ag}$ or Reed, Simon ReedSim that (for general dimensions $\mathbb{R}^{d}$ )

$$
\left\|\left(-\Delta-\left(\lambda^{2} \pm i 0\right)\right)^{-1} f\right\|_{L^{2,-\sigma}} \lesssim\|f\|_{L^{2, \sigma}}
$$

provided $\sigma>\frac{1}{2}$ and $1<\lambda<2$, say. This bound is known as the limiting absorption principle. It extends easily to $\lambda>1$ with a constant that decays like $\lambda^{-2+2 \sigma}$ (this is not optimal but sufficient for our purposes). Indeed, use that $\lambda^{d-2}(-\Delta-1)^{-1}(\lambda x)=\left(-\Delta-\lambda^{2}\right)^{-1}(x)$ for the kernels of the resolvents. Since $L^{2, \alpha}$ embeds in $L^{2, \sigma}$ for all $\alpha>\sigma$, it is to our advantage to choose $\sigma=\frac{1}{2}+$ so that

$$
\left\|\left(-\triangle-\left(\lambda^{2} \pm i 0\right)\right)^{-1} f\right\|_{L^{2,-\sigma}} \lesssim \lambda^{-1+}\|f\|_{L^{2, \sigma}}
$$

for all $\sigma>\frac{1}{2}, \lambda>1$. Throughout this paper, the notation $a+$ or $a^{+}$for some number $a$ means $a+\varepsilon$ for an arbitrarily small, but fixed $\varepsilon>0$. Similarly with $a-$ and $a^{-}$. The free resolvent $R_{0}\left(\lambda^{2} \pm i 0\right):=\left(-\Delta-\left(\lambda^{2} \pm i 0\right)\right)^{-1}$ satisfies the following well-known bounds.

Proposition 9. The derivatives $\frac{d^{j}}{d \lambda^{j}}\left[R_{0}\left(\lambda^{2} \pm i 0\right)\right]$ satisfy the uniform bounds

$$
\sup _{\lambda}\left\|\frac{d^{j}}{d \lambda^{j}}\left[R_{0}\left(\lambda^{2} \pm i 0\right)\right] f\right\|_{L^{2,-\sigma}} \lesssim\|f\|_{L^{2, \sigma}}
$$

for all $\sigma>j+\frac{1}{2}$ and $j \geq 1$.

Proof. The kernel of $\frac{d^{j}}{d \lambda^{j}}\left[R_{0}\left(\lambda^{2} \pm i 0\right)\right]$ has the explicit form

$$
\frac{d^{j}}{d \lambda^{j}}\left[R_{0}\left(\lambda^{2} \pm i 0\right)\right](x, y)=\frac{1}{4 \pi} e^{ \pm i \lambda|x-y|}|x-y|^{j-1}
$$

The Hilbert-Schmidt norm of this operator as a mapping from $L^{2, \sigma}$ to $L^{2,-\sigma}$ is given by

$$
\left\|\frac{d^{j}}{d \lambda^{j}}\left[R_{0}\left(\lambda^{2} \pm i 0\right)\right]\right\|_{H S}^{2}=C \iint_{\mathbb{R}^{6}}\langle x\rangle^{-2 \sigma}|x-y|^{2 j-2}\langle y\rangle^{-2 \sigma} d x d y
$$

The integral may be divided into the three domains $|x| \leq \frac{|y|}{2},|x-y| \leq \frac{|y|}{2}$, and the complement of these two. For a fixed point $y \in \mathbb{R}^{3}$, the respective regions contribute $\langle y\rangle^{2 j-2-2 \sigma},\langle y\rangle^{2 j+1-4 \sigma}$, and $\langle y\rangle^{2 j+1-4 \sigma}$ again when integrated with respect to $x$. If $\sigma>j+\frac{1}{2}$, each of these exponents is less than -3 , leading to a convergent integral in $d y$. Note that all dependence on $\lambda$ was removed by taking absolute values. 
Next, one transfers these estimates to $R_{V}\left(\lambda^{2} \pm i 0\right)$ by means of the resolvent identity

$$
\begin{aligned}
& R_{V}\left(\lambda^{2} \pm i 0\right)=R_{0}\left(\lambda^{2} \pm i 0\right)-R_{0}\left(\lambda^{2} \pm i 0\right) V R_{V}\left(\lambda^{2} \pm i 0\right) \\
& R_{V}\left(\lambda^{2} \pm i 0\right)=\left(I+R_{0}\left(\lambda^{2} \pm i 0\right) V\right)^{-1} R_{0}\left(\lambda^{2} \pm i 0\right)
\end{aligned}
$$

Now $S=S(\lambda):=I+R_{0}\left(\lambda^{2} \pm i 0\right) V$ is a perturbation of the identity by the compact operator $R_{0}\left(\lambda^{2} \pm i 0\right) V: L^{2,-\sigma} \rightarrow L^{2,-\sigma}$ with $\sigma>\frac{1}{2}$ provided $|V(x)| \lesssim(1+|x|)^{-1-}$. The compactness here follows from the fact that the resolvent gains two derivatives in the weighted $L^{2}$ space. Thus $S^{-1}$ exists iff $S f=0$ implies $f=0$ for any $f \in L^{2,-\sigma}$. But $S f=0$ is formally equivalent to $(-\Delta+V) f=\lambda^{2} f$. Since $\lambda>0$, it follows from Agmon $\mathrm{Ag}$ that in fact $f$ which was only assumed to be in $L^{2, \sigma}$ for every $\sigma>\frac{1}{2}$, has to be an eigenfunction (i.e., in $L^{2}$ ). But positive embedded eigenvalues do not exist by Kato's theorem, see ReedSim, Section XIII.8 for all this. Hence $S(\lambda)^{-1}: L^{2,-\sigma} \rightarrow L^{2,-\sigma}$ exists for all $\lambda>0$ provided $\sigma>\frac{1}{2}$. Furthermore, $S(\lambda)$ converges to the identity operator as $\lambda \rightarrow \infty$ which then implies that $S(\lambda)^{-1}$ is uniformly bounded for all $\lambda>\lambda_{0}$. Consequently, for $\sigma=\frac{1}{2}+$,

$$
\left\|R_{V}\left(\lambda^{2} \pm i 0\right)\right\|_{L^{2, \sigma} \rightarrow L^{2,-\sigma}} \lesssim \lambda^{-1+} .
$$

To handle derivatives of $R_{V}\left(\lambda^{2} \pm i 0\right)$, one checks that

$$
\frac{d}{d \lambda} R_{V}\left(\lambda^{2} \pm i 0\right)=-S(\lambda)^{-1} \frac{d}{d \lambda} R_{0}\left(\lambda^{2} \pm i 0\right) V S(\lambda)^{-1} R_{0}\left(\lambda^{2} \pm i 0\right)+S(\lambda)^{-1} \frac{d}{d \lambda} R_{0}\left(\lambda^{2} \pm i 0\right),
$$

and since $\sup _{\lambda>\lambda_{0}}\left\|S(\lambda)^{-1}\right\|_{L^{2,-\sigma} \rightarrow L^{2,-\sigma}}<\infty$ for $\sigma>\frac{1}{2}$, it follows that also

$$
\sup _{\lambda>\lambda_{0}}\left\|\frac{d}{d \lambda} R_{V}\left(\lambda^{2} \pm i 0\right)\right\|_{L^{2, \sigma} \rightarrow L^{2,-\sigma}} \lesssim 1 \text { for } \sigma>\frac{3}{2} .
$$

Note from (26) that one needs to assume the decay $|V(x)| \lesssim(1+|x|)^{-2-\varepsilon}$ for this to hold. Indeed, $V$ needs to take $L^{2,-\frac{1}{2}-} \rightarrow L^{2, \frac{3}{2}+}$. By a similar argument,

$$
\left\|\frac{d^{2}}{d \lambda^{2}} R_{V}\left(\lambda^{2} \pm i 0\right)\right\|_{L^{2, \sigma} \rightarrow L^{2,-\sigma}} \lesssim 1 \text { for } \sigma>\frac{5}{2} .
$$

This estimate requires the decay $|V(x)| \lesssim(1+|x|)^{-3-}$ by an analogous formula to (26).

Let $R_{0}^{ \pm}\left(\lambda^{2}\right):=R_{0}\left(\lambda^{2} \pm i 0\right)$. Moreover, set

$$
G_{ \pm, x}\left(\lambda^{2}\right)\left(x_{1}\right):=e^{\mp i \lambda|x|} R_{0}\left(\lambda^{2} \pm i 0\right)\left(x_{1}, x\right)=\frac{e^{ \pm i \lambda\left(\left|x_{1}-x\right|-|x|\right)}}{4 \pi\left|x_{1}-x\right|} .
$$

Similar kernels appear already in Yajima's work Yaj2 (see his high energy section). Removing $f, g$ from (16), we are led to proving that

$$
\begin{aligned}
& \left|\int_{0}^{\infty} e^{i t \lambda^{2}} e^{ \pm i \lambda(|x|+|y|)} \chi(\lambda / L)\left(1-\chi\left(\lambda / \lambda_{0}\right)\right) \lambda\left\langle V R_{V}^{ \pm}\left(\lambda^{2}\right) V\left(R_{0}^{ \pm}\left(\lambda^{2}\right) V\right)^{m} G_{ \pm, y}\left(\lambda^{2}\right),\left(R_{0}^{\mp}\left(\lambda^{2}\right) V\right)^{m} G_{ \pm, x}^{*}\left(\lambda^{2}\right)\right\rangle d \lambda\right| \\
& \lesssim|t|^{-\frac{3}{2}}
\end{aligned}
$$

uniformly in $x, y \in \mathbb{R}^{3}$ and $L \geq 1$. 
Proposition 10. The derivatives of $G_{+, x}\left(\lambda^{2}\right)$ satisfy the estimates

$$
\begin{aligned}
& \sup _{x \in \mathbb{R}^{3}}\left\|\frac{d^{j}}{d \lambda^{j}} G_{+, x}\left(\lambda^{2}\right)\right\|_{L^{2,-\sigma}}<C_{j, \sigma} \text { provided } \sigma>\frac{1}{2}+j \\
& \sup _{x \in \mathbb{R}^{3}}\left\|\frac{d^{j}}{d \lambda^{j}} G_{+, x}\left(\lambda^{2}\right)\right\|_{L^{2,-\sigma}}<\frac{C_{j, \sigma}}{\langle x\rangle} \text { provided } \sigma>\frac{3}{2}+j
\end{aligned}
$$

for all $j \geq 0$.

Proof. This follows from the explicit formula

$$
\begin{aligned}
\left\|\frac{d^{j}}{d \lambda^{j}} \frac{e^{i \lambda(|u-x|-|x|)}}{|x-u|}\langle u\rangle^{-\sigma} d u\right\|_{2} & =\left(\int_{\mathbb{R}^{3}} \frac{(|u-x|-|x|)^{2 j}}{|x-u|^{2}}\langle u\rangle^{-2 \sigma} d u\right)^{\frac{1}{2}} \\
& \leq\left(\int_{\mathbb{R}^{3}} \frac{\langle u\rangle^{2(j-\sigma)}}{|x-u|^{2}} d u\right)^{\frac{1}{2}}
\end{aligned}
$$

The final estimate on this integral is obtained by dividing $\mathbb{R}^{3}$ into the regions $|u|<\frac{|x|}{2},|x-u|<\frac{|x|}{2}$, and the complement of these two. If $\frac{1}{2}<(\sigma-j)<\frac{3}{2}$, then each of these regions contributes $\langle x\rangle^{\frac{1}{2}+j-\sigma}$ to the total. If $\sigma>\frac{3}{2}+j$, the first region instead contributes $\langle x\rangle^{-1}$, making it the dominant term.

Rewrite the integral in (28) in the form (with $L=\infty$ )

$$
I^{ \pm}(t, x, y):=\int_{0}^{\infty} e^{i t \lambda^{2} \pm i \lambda(|x|+|y|)} a_{x, y}^{ \pm}(\lambda) d \lambda .
$$

Then in view of (24), (25), (27), and Propositions 9 and 10, one concludes that $a_{x, y}^{ \pm}(\lambda)$ has two derivatives in $\lambda$ and

$$
\begin{aligned}
\left|\frac{d^{j}}{d \lambda^{j}} a_{x, y}^{ \pm}(\lambda)\right| \lesssim(1+\lambda)^{-2^{+}}(\langle x\rangle\langle y\rangle)^{-1} \text { for } j=0,1, \text { and all } \lambda>1 \\
\left|\frac{d^{2}}{d \lambda^{2}} a_{x, y}^{ \pm}(\lambda)\right| \lesssim(1+\lambda)^{-2^{+}} \quad \text { for all } \lambda>1,
\end{aligned}
$$

which in particular justifies taking $L=\infty$ in (30). This requires that one takes $m$ sufficiently large ( $m=2$ is sufficient) and that $|V(x)| \lesssim(1+|x|)^{-\beta}$ for some $\beta>3$. The latter condition arises as follows: Consider, for example, the case where two derivatives fall one of the $G$-terms at the ends. Then $V$ has to compensate for $\frac{5}{2}^{+}$powers because of (29), and also a $\frac{1}{2}^{+}$power from

$$
\left\|R_{0}^{ \pm}\left(\lambda^{2}\right) f\right\|_{L^{2,-\frac{1}{2}-}} \lesssim \lambda^{-1^{+}}\|f\|_{L^{2, \frac{1}{2}+}} .
$$

Similarly with the other terms.

As far as $I^{+}(t, x, y)$ is concerned, note that on the support of $a_{x, y}^{ \pm}(\lambda)$ the phase $t \lambda^{2}+\lambda(|x|+|y|)$ has no critical point. Two integrations by parts yield the bound $\left|I^{+}(t, x, y)\right| \lesssim t^{-2}$.

In the case of $I^{-}(t, x, y)$ the phase $t \lambda^{2}-\lambda(|x|+|y|)$ has a unique critical point at $\lambda_{1}=(|x|+|y|) /(2 t)$. If $\lambda_{1} \ll \lambda_{0}$, then two integration by parts again yield a bound of $t^{-2}$. If $\lambda_{1} \gtrsim \lambda_{0}$ then the bound $\max (|x|,|y|) \gtrsim t$ is also true, and stationary phase contributes $t^{-\frac{1}{2}}(\langle x\rangle\langle y\rangle)^{-1} \lesssim t^{-\frac{3}{2}}$, as desired. 
Strictly speaking, these estimates are only useful when $t>1$. On the other hand, when $0<t<1$ there is nothing to prove since $I^{ \pm}(t, x, y) \lesssim 1$ by (31).

To apply stationary phase properly, one should restrict $a_{x, y}^{ \pm}(\lambda)$ to a compact interval of the form $\left[\lambda_{1}-C, \lambda_{1}+C\right]$ for some constant $C \gg 1$. Outside of this interval, one uses the decay given by (31) in terms of $\lambda$. Two integrations by parts yield the bound $t^{-3}$ for the remaining piece of $I^{-}(t, x, y)$. This concludes the high-energy part of the argument.

\subsection{Low energies}

In view of (16) and (18) it remains to control the low-energy part

$$
\begin{aligned}
& \left\langle e^{i t H} \chi\left(\sqrt{H} / \lambda_{0}\right) P_{a . c .} f, g\right\rangle \\
& =\int_{0}^{\infty} e^{i t \lambda^{2}} \lambda \chi\left(\lambda / \lambda_{0}\right)\left\langle\left[R_{V}\left(\lambda^{2}+i 0\right)-R_{V}\left(\lambda^{2}-i 0\right)\right] f, g\right\rangle \frac{d \lambda}{\pi i}
\end{aligned}
$$

If $f, g \in L^{1}$, this can be done by evaluating the supremum

$$
\sup _{\mathbf{x}, \mathbf{y} \in \mathbb{R}^{3}}\left|\int_{0}^{\infty} e^{i t \lambda^{2}} \lambda \chi\left(\lambda / \lambda_{0}\right)\left[R_{V}^{+}\left(\lambda^{2}\right)-R_{V}^{-}\left(\lambda^{2}\right)\right](\mathbf{x}, \mathbf{y}) d \lambda\right|
$$

We will use the resolvent identity

$$
R_{V}^{ \pm}\left(\lambda^{2}\right)=R_{0}^{ \pm}\left(\lambda^{2}\right)-R_{0}^{ \pm}\left(\lambda^{2}\right) V\left(I+R_{0}^{ \pm}\left(\lambda^{2}\right) V\right)^{-1} R_{0}^{ \pm}\left(\lambda^{2}\right)
$$

The resolvents $R_{0}^{ \pm}\left(\lambda^{2}\right)$ have an explicit kernel representation

$$
R_{0}^{ \pm}\left(\lambda^{2}\right)(\mathbf{x}, \mathbf{y})=\frac{e^{ \pm i \lambda|\mathbf{x}-\mathbf{y}|}}{4 \pi|\mathbf{x}-\mathbf{y}|}
$$

The numerator of this expression always has complex magnitude 1 , therefore the size of $\left|R_{0}^{ \pm}\left(\lambda^{2}\right)\right|$ does not depend on $\lambda$. We will now estimate the Hilbert-Schmidt norm of $R_{0}^{ \pm}\left(\lambda^{2}\right)$ as a linear map between the weighted spaces $L^{2, \sigma}$ and $L^{2,-\alpha}$. Let

$$
\|R\|_{H S(\sigma,-\alpha)}^{2}=\iint_{\mathbb{R}^{6}}\langle\mathbf{x}\rangle^{-2 \sigma}|R(\mathbf{x}, \mathbf{y})|^{2}\langle\mathbf{y}\rangle^{-2 \alpha} d \mathbf{x} d \mathbf{y}
$$

denote this norm. The following proposition is a well-known bound on the free resolvents.

Proposition 11. If $\sigma, \alpha>\frac{1}{2}$, and $\sigma+\alpha>2$, then

$$
\sup _{\lambda}\left\|R_{0}^{ \pm}\left(\lambda^{2}\right)\right\|_{H S(\sigma,-\alpha)} \leq C_{\sigma, \alpha}
$$

Proof. The integral

$$
\iint_{\mathbb{R}^{6}}\langle\mathbf{x}\rangle^{-2 \sigma} \frac{1}{|\mathbf{x}-\mathbf{y}|^{2}}\langle\mathbf{y}\rangle^{-2 \alpha} d \mathbf{x} d \mathbf{y}
$$

may be broken up into three disjoint domains:

Domain 1: $|\mathbf{x}| \leq \frac{1}{2}|\mathbf{y}|$, which requires $|\mathbf{x}-\mathbf{y}| \sim|\mathbf{y}|$. The integral over Domain 1 contributes less than $\int_{\mathbb{R}^{3}}\langle\mathbf{y}\rangle^{3-2 \sigma}\langle\mathbf{y}\rangle^{-1-2 \alpha} d \mathbf{y}$, which is bounded by a constant $C_{\sigma, \alpha}$, to the total integral. 
Domain 2: $|\mathbf{x}-\mathbf{y}| \leq \frac{1}{2}|\mathbf{y}|$, which requires $|\mathbf{x}| \sim|\mathbf{y}|$. The integral over Domain 2 contributes less than $\int_{\mathbb{R}^{3}}|y|\langle\mathbf{y}\rangle^{-2 \sigma-2 \alpha} d \mathbf{y}$, which is also bounded by $C_{\sigma, \alpha}$, to the total integral.

Domain 3: $|\mathbf{x}|,|\mathbf{x}-\mathbf{y}| \geq \frac{1}{2}|\mathbf{y}|$, which requires $|\mathbf{x}| \sim|\mathbf{x}-\mathbf{y}|$. The integral over Domain 3 contributes less than $\int_{\mathbb{R}^{3}}\langle\mathbf{y}\rangle^{1-2 \sigma}\langle\mathbf{y}\rangle^{-2 \alpha} d \mathbf{y} \lesssim C_{\sigma, \alpha}$ to the total integral.

If $|V(\mathbf{x})| \lesssim\langle\mathbf{x}\rangle^{-\beta}$ for some $\beta>3$, it follows that the operator $R_{0}^{ \pm}\left(\lambda^{2}\right) V$ is compact on the weighted space $L^{2, \sigma}\left(\mathbb{R}^{3}\right)$ for all choices of $-\frac{5}{2} \leq \sigma<-\frac{1}{2}$. Indeed, one checks by means of Proposition [1] that $R_{0}^{ \pm}\left(\lambda^{2}\right) V$ maps $L^{2, \sigma}\left(\mathbb{R}^{3}\right)$ compactly into $L^{2, \sigma+1}\left(\mathbb{R}^{3}\right)$ for all $\sigma \in\left[-\frac{5}{2},-\frac{3}{2}\right)$.

Let $S_{0}=I+R_{0}(0) V$. By compactness of $R_{0}(0) V$, the invertibility of $S_{0}$ depends only on whether a solution exists in $L^{2, \sigma}$ to the equation $\psi=-R_{0}(0) V \psi$. However if a solution $\psi$ satisfies $\psi \in L^{2, \sigma}$ for some $\sigma \geq-\frac{5}{2}$, then $\psi=-R_{0}(0) V \psi \in L^{2, \alpha}$ for any choice of $\alpha<-\frac{3}{2}$. Applying the bootstrapping process again, we see that the solution $\psi$ must lie in $L^{2, \alpha}$ for all $\alpha<-\frac{1}{2}$.

It is easy to see that this same function $\psi$ is also a distributional solution to $(\triangle+V) \psi=0$. Conversely, any distributional solution of $(\triangle+V) \psi=0$ with $\psi \in L^{2,-\frac{1}{2}-}$ satisfies $S_{0} \psi=0$. It follows that $S_{0}$ is invertible in $L^{2, \sigma},-\frac{5}{2} \leq \sigma<-\frac{1}{2}$ precisely when zero energy is neither an eigenvalue nor a resonance of the potential $V$, see Definition 6 .

Write $R_{0}^{ \pm}\left(\lambda^{2}\right)=R_{0}(0)+B^{ \pm}(\lambda)$. Then

$$
\left[I+R_{0}^{ \pm}\left(\lambda^{2}\right) V\right]^{-1}=S_{0}^{-1}\left[I+B^{ \pm}(\lambda) V S_{0}^{-1}\right]^{-1}
$$

Examining the kernel,

$$
B^{ \pm}(\lambda)(\mathbf{x}, \mathbf{y})=\frac{e^{ \pm i \lambda|\mathbf{x}-\mathbf{y}|}-1}{4 \pi|\mathbf{x}-\mathbf{y}|}
$$

which satisfies the size estimates

$$
\left|B^{ \pm}(\lambda)(\mathbf{x}, \mathbf{y})\right| \lesssim\left\{\begin{array}{cc}
\lambda & \text { if }|\mathbf{x}-\mathbf{y}| \leq 1 / \lambda \\
|\mathbf{x}-\mathbf{y}|^{-1} & \text { if }|\mathbf{x}-\mathbf{y}| \geq 1 / \lambda
\end{array}\right.
$$

The first $\lambda$-derivative of $B^{ \pm}$has kernel $\left(B^{ \pm}\right)^{\prime}(\lambda)(\mathbf{x}, \mathbf{y})=\frac{ \pm i}{4 \pi} e^{ \pm i \lambda|\mathbf{x}-\mathbf{y}|}$ with the obvious bound $\left|\left(B^{ \pm}\right)^{\prime}(\lambda)(\mathbf{x}, \mathbf{y})\right| \leq C$.

The symmetry between $B^{+}$and $B^{-}$is expressed by the relationship

$$
B^{-}(\lambda)=B^{+}(-\lambda) \text { for all } \lambda \geq 0
$$

Proposition 12. If $\sigma, \alpha>\frac{1}{2}$, and $\sigma+\alpha>2$, then $\lim _{\lambda \rightarrow 0}\left\|B^{ \pm}(\lambda)\right\|_{H S(\sigma,-\alpha)}=0$.

Proof. The kernels $B^{ \pm}(\lambda)(\mathbf{x}, \mathbf{y})$ are pointwise dominated by $\frac{1}{|\mathbf{x}-\mathbf{y}|}$, which has a finite $H S(\sigma,-\alpha)$ norm by Proposition 11 The result then follows from the dominated convergence theorem.

Corollary 13. If $|V(x)| \lesssim\langle x\rangle^{-\beta}$ for some choice of $\beta>3$, then

$$
\lim _{\lambda \rightarrow 0}\left\|B^{ \pm}(\lambda) V S_{0}^{-1}\right\|_{H S(\sigma, \sigma)}=0
$$

for all $\sigma \in\left(-\frac{5}{2},-\frac{1}{2}\right)$.

Proof. One has $V S_{0}^{-1}: L^{2, \sigma} \rightarrow L^{2, \sigma+3+}$ provided that $-\frac{5}{2}<\sigma<-\frac{1}{2}$. The proposition implies that $\left\|B^{ \pm}(\lambda)\right\|_{H S(\sigma+3+, \sigma)} \rightarrow 0$ as $\lambda \rightarrow 0$. 
Claim: $\left\|\left(B^{+}\right)^{\prime}(\lambda)\right\|_{H S(\sigma,-\alpha)} \leq C$ if $\sigma, \alpha>\frac{3}{2}$.

Proof. This is trivial because the function $\langle\mathbf{x}\rangle^{-2 \sigma}\langle\mathbf{y}\rangle^{-2 \alpha}$ is integrable over $\mathbb{R}^{6}$.

For sufficiently small $\lambda<\lambda_{0}$, it is then possible to expand

$$
\tilde{B}^{ \pm}(\lambda)=\left[I+B^{ \pm}(\lambda) V S_{0}^{-1}\right]^{-1}
$$

as a Neumann series in the norm $\|\cdot\|_{H S(\sigma, \sigma)}$ for all values $-\frac{5}{2}<\sigma<-\frac{1}{2}$.

The symmetry $\tilde{B}^{-}(\lambda)=\tilde{B}^{+}(-\lambda)$ is still valid.

For ease of notation, define $\chi_{0}(\lambda)=\chi\left(\lambda / \lambda_{0}\right)$ and $\chi_{1}(\lambda)=\chi\left(\lambda / 2 \lambda_{0}\right)$. Note that $\chi_{1} \chi_{0}=\chi_{0}$. In view of (33) and (34) we wish to control the size of

$$
\begin{aligned}
& \sup _{\mathbf{x}, \mathbf{y} \in \mathbb{R}^{3}} \mid \int_{0}^{\infty} e^{i t \lambda^{2}} \lambda \chi_{0}(\lambda)\left[\left[R_{0}^{+}\left(\lambda^{2}\right)-R_{0}^{-}\left(\lambda^{2}\right)\right]\right. \\
& \left.\quad-\left[R_{0}^{+}\left(\lambda^{2}\right) V S_{0}^{-1} \tilde{B}^{+}(\lambda) R_{0}^{+}\left(\lambda^{2}\right)-R_{0}^{-}\left(\lambda^{2}\right) V S_{0}^{-1} \tilde{B}^{-}(\lambda) R_{0}^{-}\left(\lambda^{2}\right)\right]\right](\mathbf{x}, \mathbf{y}) d \lambda \mid \\
& \leq \sup _{\mathbf{x}, \mathbf{y} \in \mathbb{R}^{3}}\left|\int_{-\infty}^{\infty} e^{i t \lambda^{2}} \lambda \chi_{0}(\lambda) \frac{e^{i \lambda|\mathbf{x}-\mathbf{y}|}}{4 \pi|\mathbf{x}-\mathbf{y}|} d \lambda\right| \\
& \quad+\sup _{\mathbf{x}, \mathbf{y} \in \mathbb{R}^{3}}\left|\int_{-\infty}^{\infty} e^{i t \lambda^{2}} \lambda \iint_{\mathbb{R}^{6}} \frac{V\left(x_{4}\right) e^{i \lambda\left|\mathbf{y}-x_{4}\right|}}{\left|\mathbf{y}-x_{4}\right|}\left(S_{0}^{-1}\left(\chi_{0} \tilde{B}^{+}\right)(\lambda)\left(x_{4}, x_{1}\right)\right) \frac{e^{i \lambda\left|\mathbf{x}-x_{1}\right|}}{\left|\mathbf{x}-x_{1}\right|} d x_{1} d x_{4} d \lambda\right|
\end{aligned}
$$

The first term is simply the low-energy part of the free Schrödinger evolution, which is known to be dispersive.

The second term can be integrated by parts once, leaving

$$
\sup _{\mathbf{x}, \mathbf{y} \in \mathbb{R}^{3}} \frac{1}{2 t}\left|\int_{-\infty}^{\infty} e^{i t \lambda^{2}} \iint_{\mathbb{R}^{6}} \frac{d}{d \lambda}\left[\frac{V\left(x_{4}\right) e^{i \lambda\left|\mathbf{y}-x_{4}\right|}}{\left|\mathbf{y}-x_{4}\right|}\left(S_{0}^{-1}\left(\chi_{0} \tilde{B}^{+}\right)(\lambda)\left(x_{4}, x_{1}\right)\right) \frac{e^{i \lambda\left|\mathbf{x}-x_{1}\right|}}{\left|\mathbf{x}-x_{1}\right|}\right] d x_{1} d x_{4} d \lambda\right|
$$

to be controlled. Consider the term where $\frac{d}{d \lambda}$ falls on $\tilde{B}^{+}(\lambda)$. The others will be similar.

Using Parseval's identity, and the fact that $\left\|\left(e^{i t(\cdot)^{2}}\right)^{\wedge}(u)\right\|_{L^{\infty}(u)}=C t^{-1 / 2}$, this is less than

$$
\sup _{\mathbf{x}, \mathbf{y} \in \mathbb{R}^{3}} \frac{1}{t^{3 / 2}} \int_{-\infty}^{\infty}\left|\iint_{\mathbb{R}^{6}} \frac{V\left(x_{4}\right)}{\left|\mathbf{y}-x_{4}\right|} S_{0}^{-1}\left[\chi_{0}\left(\tilde{B}^{+}\right)^{\prime}\right]^{\vee}\left(u+\left|\mathbf{y}-x_{4}\right|+\left|\mathbf{x}-x_{1}\right|\right)\left(x_{4}, x_{1}\right) \frac{1}{\left|\mathbf{x}-x_{1}\right|} d x_{1} d x_{4}\right| d u
$$

If the absolute value is taken inside the inner integral, then Fubini's theorem may be used to exchange the order of integration to obtain

$$
\begin{array}{r}
\sup _{\mathbf{x}, \mathbf{y} \in \mathbb{R}^{3}} \frac{1}{t^{3 / 2}} \iint_{\mathbb{R}^{6}} \int_{-\infty}^{\infty} \frac{\left|V\left(x_{4}\right)\right|}{\left|\mathbf{y}-x_{4}\right|}\left|S_{0}^{-1}\left[\chi_{0}\left(\tilde{B}^{+}\right)^{\prime}\right]^{\vee}\left(u+\left|\mathbf{y}-x_{4}\right|+\left|\mathbf{x}-x_{1}\right|\right)\left(x_{4}, x_{1}\right)\right| \frac{1}{\left|\mathbf{x}-x_{1}\right|} d u d x_{1} d x_{4} \\
\leq \sup _{\mathbf{x}, \mathbf{y} \in \mathbb{R}^{3}} \frac{1}{t^{3 / 2}}\left\|\frac{|V(\cdot)|}{|\mathbf{y}-\cdot|}\right\|_{L^{2,2^{+}}}\left\|\int\left|S_{0}^{-1}\left[\chi_{0}\left(\tilde{B}^{+}\right)^{\prime}\right]^{\vee}(u)\right| d u\right\|_{H S\left(-1^{-},-2^{-}\right)}\left\||\mathbf{x}-\cdot|^{-1}\right\|_{L^{2,-1^{-}}}
\end{array}
$$


The weighted $L^{2,-1^{-}}\left(d x_{1}\right)$-norm of $\left|\mathbf{x}-x_{1}\right|^{-1}$ is uniformly bounded for all choices of $\mathbf{x} \in \mathbb{R}^{3}$. In fact, these functions are even bounded in the weaker $L^{2, \sigma}$ norm for any $\sigma<-\frac{1}{2}$. Similarly, the functions $\frac{V\left(x_{4}\right)}{\left|\mathbf{y}-x_{4}\right|}$ are uniformly bounded in $L^{2, \sigma}\left(d x_{4}\right)$ for any $\sigma<\beta-\frac{1}{2}$. We are assuming $\beta>3$, which is more than sufficient. It therefore remains only to control the size of

$$
\left\|\int\left|S_{0}^{-1}\left[\chi_{0}\left(\tilde{B}^{+}\right)^{\prime}\right]^{\vee}(u)\right| d u\right\|_{H S\left(-1^{-},-2^{-}\right)}
$$

Minkowski's Inequality allows us to bring the norm inside the integral. Recall that $S_{0}^{-1}$ is a bounded operator on $L^{2,-2^{-}}$, and that the composition of a bounded operator and a Hilbert-Schmidt operator is also Hilbert-Schmidt. The problem then reduces to establishing existence of a number $\lambda_{0}>0$ such that

$$
\int_{-\infty}^{\infty}\left\|\left[\chi_{0}\left(\tilde{B}^{+}\right)^{\prime}\right]^{\vee}(u)\right\|_{H S\left(-1^{-},-2^{-}\right)} d u<\infty
$$

The operators $\tilde{B}^{+}(\lambda)$ were originally defined by the convergent Neumann series

$$
\tilde{B}^{+}(\lambda)=\left[I+B^{+}(\lambda) V S_{0}^{-1}\right]^{-1}=\sum_{n=0}^{\infty}\left(-B^{+}(\lambda) V S_{0}^{-1}\right)^{n}
$$

Thus

$$
\chi_{0}(\lambda)\left(\tilde{B}^{+}\right)^{\prime}(\lambda)=\sum_{n=1}^{\infty} \sum_{m=0}^{n-1}(-1)^{n}\left(\left(\chi_{1} B^{+}\right)(\lambda) V S_{0}^{-1}\right)^{m} \chi_{0}(\lambda)\left(B^{+}\right)^{\prime}(\lambda) V S_{0}^{-1}\left(\left(\chi_{1} B^{+}\right)(\lambda) V S_{0}^{-1}\right)^{n-(m+1)}
$$

We will take the Fourier transform of $\chi_{0}\left(\tilde{B}^{+}\right)^{\prime}$ term-wise and determine that the resulting series is convergent in the norm $L^{1}\left(d u ; H S\left(-1^{-},-2^{-}\right)\right)$. The following refinement of Proposition 12 is especially useful.

Proposition 14. Suppose $\sigma, \alpha>\frac{1}{2}$, and $\alpha+\sigma>2$. Let $K(\lambda)$ be an integral operator on $\mathbb{R}^{3}$ whose kernel $K(\lambda)(\mathbf{x}, \mathbf{y})$ satisfies the size estimates in (35). Then

$$
\|K(\lambda)\|_{H S(\sigma,-\alpha)} \leq C_{\sigma, \alpha, \gamma}|\lambda|^{\gamma}
$$

for any $\gamma<\min \left(\sigma+\alpha-2, \sigma-\frac{1}{2}, \alpha-\frac{1}{2}, 1\right)$. Equality is possible in the choice of $\gamma$ provided $\sigma, \alpha \neq \frac{3}{2}$.

Proof. The size conditions in (35) guarantees that

$$
\|K(\lambda)\|_{H S(\sigma,-\alpha)}^{2} \lesssim \lambda^{2} \iint_{\left\{|\mathbf{x}-\mathbf{y}|<\frac{1}{\lambda}\right\}}\langle\mathbf{x}\rangle^{-2 \sigma}\langle\mathbf{y}\rangle^{-2 \alpha} d \mathbf{x} d \mathbf{y}+\iint_{\left\{|\mathbf{x}-\mathbf{y}|>\frac{1}{\lambda}\right\}}\langle\mathbf{x}\rangle^{-2 \sigma} \frac{1}{|\mathbf{x}-\mathbf{y}|^{2}}\langle\mathbf{y}\rangle^{-2 \alpha} d \mathbf{x} d \mathbf{y}
$$

The first of these integrals is broken up into two domains:

Domain 1: $\max (|\mathbf{x}|,|\mathbf{y}|) \leq \frac{3}{\lambda}$.

Domain 2: $|\mathbf{y}|>\frac{2}{\lambda}, \mathbf{x} \in B\left(\mathbf{y}, \frac{1}{\lambda}\right)$, which also requires that $|\mathbf{x}| \sim|\mathbf{y}|$.

The second integral is broken up into four domains, namely:

Domain 3: $\left\{|\mathbf{x}-\mathbf{y}| \leq \frac{1}{2}|\mathbf{y}|\right\}$, which requires $|\mathbf{y}|>\frac{2}{\lambda}$ and $|\mathbf{x}| \sim|\mathbf{y}|$.

Domain 4: $\left\{|\mathbf{x}| \leq \frac{1}{2}|\mathbf{y}|\right\}$, which requires $|\mathbf{y}|>\frac{2}{3 \lambda}$ and $|\mathbf{x}-\mathbf{y}| \sim|\mathbf{y}|$. 
Domain 5: $\left\{|\mathbf{x}|,|\mathbf{x}-\mathbf{y}|>\frac{1}{2}|\mathbf{y}| ;|\mathbf{y}|>\frac{2}{\lambda}\right\}$, which requires $|\mathbf{x}| \sim|\mathbf{x}-\mathbf{y}|$.

Domain 6: $\left\{|\mathbf{x}|,|\mathbf{x}-\mathbf{y}|>\frac{1}{2}|\mathbf{y}| ;|\mathbf{y}| \leq \frac{2}{\lambda}\right\}$, which requires $|\mathbf{x}| \sim|\mathbf{x}-\mathbf{y}|$. In this domain, only values $|\mathbf{x}-\mathbf{y}|>\frac{1}{\lambda}>\frac{|\mathbf{y}|}{2}$ can make a nonzero contribution.

With the given restrictions on $\sigma$ and $\alpha$ to insure finiteness of each integral, Domain 1 contributes no more than $C \lambda^{2 \gamma}$ to the total. Each of the other domains contributes $C \lambda^{2 \gamma_{i}}$, where $\gamma_{i}$ is one of the four possible exponents in the definition of $\gamma$.

Lemma 15. The Fourier transform of $\chi_{0}\left(B^{+}\right)^{\prime}$ in the variable $\lambda$ satisfies the property

$$
\int_{-\infty}^{\infty}\left\|\left[\chi_{0}\left(B^{+}\right)^{\prime}\right]^{\vee}(u)\right\|_{H S\left(2^{+},-2^{-}\right)} d u<C<\infty
$$

uniformly as $\lambda_{0} \rightarrow 0$.

Proof. First observe that for any pair of points $(\mathbf{x}, \mathbf{y}),\left[\left(B^{+}\right)^{\prime}\right]^{\vee}(u)(\mathbf{x}, \mathbf{y})=\delta(u+|\mathbf{x}-\mathbf{y}|)$, therefore

$$
\left[\chi_{0}\left(B^{+}\right)^{\prime}\right]^{\vee}(u)(\mathbf{x}, \mathbf{y})=\chi_{0}^{\vee}(u+|\mathbf{x}-\mathbf{y}|) \lesssim \lambda_{0}\left\langle\lambda_{0}(u+|\mathbf{x}-\mathbf{y}|)\right\rangle^{-10}
$$

The Hilbert-Schmidt norm is bounded above by

$$
\left\|\left[\chi_{0}\left(B^{+}\right)^{\prime}\right]^{\vee}(u)\right\|_{H S\left(2^{+},-2^{-}\right)}^{2} \lesssim \lambda_{0}^{2} \iint_{\mathbb{R}^{6}}\left\langle\lambda_{0}(u-|\mathbf{x}-\mathbf{y}|)\right\rangle^{-20}\langle\mathbf{y}\rangle^{-4^{-}}\langle\mathbf{x}\rangle^{-4^{-}} d \mathbf{x} d \mathbf{y}
$$

This is most easily evaluated via the inequality

$$
\int_{|\mathbf{x}-\mathbf{y}|=\rho}\langle\mathbf{x}\rangle^{-2 \sigma} d \mathbf{x} \lesssim\langle\rho-|\mathbf{y}|\rangle^{2-2 \sigma}
$$

for $\sigma>1$. Integrating with respect to $d \mathbf{x}$ over a spherical shell centered at $\mathbf{y}$,

$$
\begin{aligned}
\left\|\left[\chi_{0}\left(B^{+}\right)^{\prime}\right]^{\vee}(u)\right\|_{H S\left(2^{+},-2^{-}\right)}^{2} \lesssim & \lambda_{0}^{2} \int_{0}^{\infty} \int_{\mathbb{R}^{3}}\left\langle\lambda_{0}(u-\rho)\right\rangle^{-20}\langle\mathbf{y}\rangle^{-4^{-}}\langle|\mathbf{y}|-\rho\rangle^{-2^{-}} d \mathbf{y} d \rho \\
& \lesssim \lambda_{0}^{2} \int_{0}^{\infty}\left\langle\lambda_{0}(u-\rho)\right\rangle^{-20}\langle\rho\rangle^{-2^{-}} d \rho
\end{aligned}
$$

which leads to the bounds

$$
\left\|\left[\chi_{0}\left(B^{+}\right)^{\prime}\right]^{\vee}(u)\right\|_{H S\left(2^{+},-2^{-}\right)} \lesssim\left\{\begin{aligned}
\lambda_{0}, & \text { if } u \leq \frac{2}{\lambda_{0}} \\
\lambda_{0}^{-9} u^{-10}+\lambda_{0}^{1 / 2} u^{-1^{-}}, & \text {if } u \geq \frac{2}{\lambda_{0}}
\end{aligned}\right.
$$

Integrating this expression yields the quantity $C\left(1+\lambda_{0}^{1 / 2+}\right)$, which is uniformly bounded as $\lambda_{0} \rightarrow 0$.

Lemma 16. The Fourier transform of $\left(\chi_{0} B^{+}\right)$in the variable $\lambda$ satisfies the following properties:

$$
\begin{gathered}
\int_{-\infty}^{\infty}\left\|\left(\chi_{0} B^{+}\right)^{\vee}(u)\right\|_{H S\left(\frac{3}{2}^{+},-^{-}\right)} d u<C \lambda_{0}^{1 / 2+} \\
\int_{-\infty}^{\infty}\left\|\left(\chi_{0} B^{+}\right)^{\vee}(u)\right\|_{H S\left(1^{+},-\frac{3}{2}^{-}\right)} d u<C \lambda_{0}^{1 / 2+}
\end{gathered}
$$

Identical statements are also true with $\chi_{0}$ replaced by $\chi_{1}$. 
Proof. First observe that for any pair of points $(\mathbf{x}, \mathbf{y}),\left(B^{+}\right)^{\vee}(u)(\mathbf{x}, \mathbf{y})=\frac{1}{|\mathbf{x}-\mathbf{y}|}[\delta(u+|\mathbf{x}-\mathbf{y}|)-\delta(u)]$, therefore

$$
\left[\chi_{0} B^{+}\right]^{\vee}(u)(\mathbf{x}, \mathbf{y})=\frac{\chi_{0}^{\vee}(u+|\mathbf{x}-\mathbf{y}|)-\chi_{0}^{\vee}(u)}{|\mathbf{x}-\mathbf{y}|}
$$

In the case $|u| \leq \frac{2}{\lambda_{0}}$,

$$
\left|\left[\chi_{0} B^{+}\right]^{\vee}(u)(\mathbf{x}, \mathbf{y})\right| \lesssim\left\{\begin{aligned}
\lambda_{0}^{2}, & \text { if }|\mathbf{x}-\mathbf{y}| \leq \frac{1}{\lambda_{0}} \\
\frac{\lambda_{0}}{|\mathbf{x}-\mathbf{y}|}, & \text { if }|\mathbf{x}-\mathbf{y}| \geq \frac{1}{\lambda_{0}}
\end{aligned}\right.
$$

where the first estimate comes from the Mean Value theorem. Up to a factor of $\lambda_{0}$, this kernel satisfies the hypotheses of Proposition 14, with the conclusion

$$
\left\|\left[\chi_{0} B^{+}\right]^{\vee}(u)\right\|_{H S\left(\frac{3}{2}^{+},-1^{-}\right)} \lesssim\left(\lambda_{0}\right)^{3 / 2+}
$$

for all $|u| \leq \frac{2}{\lambda_{0}}$. In the case $|u| \geq \frac{2}{\lambda_{0}}$, we use the fact that $\left|\chi_{0}^{\vee}(u)\right| \leq \lambda_{0}\left\langle\lambda_{0} u\right\rangle^{-10}$ to obtain the pointwise bounds

$$
\left|\left[\chi_{0} B^{+}\right]^{\vee}(u)(\mathbf{x}, \mathbf{y})\right| \lesssim\left\{\begin{array}{cl}
\frac{1}{\lambda_{0}^{8}|u|^{10}}, & \text { if }|\mathbf{x}-\mathbf{y}| \leq \frac{|u|}{2} \\
\frac{1}{\lambda_{0}^{8}|u|^{9}|\mathbf{x}-\mathbf{y}|}, & \text { if }|\mathbf{x}-\mathbf{y}| \geq 2|u| \\
\frac{\lambda_{0}}{|u|}\left\langle\lambda_{0}(u+|\mathbf{x}-\mathbf{y}|)\right\rangle^{-10}, & \text { if } \frac{|u|}{2}<|\mathbf{x}-\mathbf{y}|<2|u|
\end{array}\right.
$$

The restriction of this kernel to the domain $\{|\mathbf{x}-\mathbf{y}| \geq 2|u|\} \cup\left\{|\mathbf{x}-\mathbf{y}| \leq \frac{|u|}{2}\right\}$ must have $H S\left(\frac{3}{2}^{+},-1^{-}\right)-$ norm of $\lambda_{0}^{-8}|u|^{-9.5^{-}}$, also by Proposition 14. Since we are assuming $|u| \geq \frac{2}{\lambda_{0}}$, this is less than $|u|^{-1.5^{-}}$. To estimate the Hilbert-Schmidt norm of the remaining annular piece, we once again use the inequality

(39)

$$
\int_{|\mathbf{x}-\mathbf{y}|=\rho}\langle\mathbf{x}\rangle^{-2 \sigma} d \mathbf{x} \lesssim\langle\rho-|\mathbf{y}|\rangle^{2-2 \sigma}
$$

for $\sigma>1$. Thus

$$
\begin{aligned}
& \frac{\lambda_{0}^{2}}{|u|^{2}} \iint_{|\mathbf{x}-\mathbf{y}| \sim|u|}\langle\mathbf{x}\rangle^{-3^{-}}\left\langle\lambda_{0}(u+|\mathbf{x}-\mathbf{y}|)\right\rangle^{-20}\langle\mathbf{y}\rangle^{-2^{-}} d \mathbf{x} d \mathbf{y} \\
& \lesssim \frac{\lambda_{0}^{2}}{|u|^{2}} \int_{\frac{|u|}{2}}^{2|u|}\left\langle\lambda_{0}(u+\rho)\right\rangle^{-20} \int_{\mathbb{R}^{3}}\langle\rho-|\mathbf{y}|\rangle^{-1^{-}}\langle\mathbf{y}\rangle^{-2^{-}} d \mathbf{y} d \rho \\
& \lesssim \frac{\lambda_{0}^{2}}{|u|^{2}} \int_{\frac{|u|}{2}}^{2|u|}\langle\rho\rangle^{-\epsilon}\left\langle\lambda_{0}(u+\rho)\right\rangle^{-20} d \rho \lesssim \frac{\lambda_{0}}{|u|^{2+\epsilon}}
\end{aligned}
$$

Putting the pieces together, it follows that

$$
\left\|\left[\chi_{0} B^{+}\right]^{\vee}\right\|_{H S\left(\frac{3}{2}{ }^{+},-1^{-}\right)} \lesssim\left(\lambda_{0}\right)^{3 / 2+\epsilon}\left\langle\lambda_{0}|u|\right\rangle^{-1-\epsilon}
$$

proving the first claim of the lemma. The second line of equation (40) follows from symmetry in the variables $\mathbf{x}$ and $\mathbf{y}$. 
Recall from equation (38) that

$$
\chi_{0}(\lambda)\left(\tilde{B}^{+}\right)^{\prime}(\lambda)=\sum_{n=1}^{\infty} \sum_{m=0}^{n-1}(-1)^{n}\left(\left(\chi_{1} B^{+}\right)(\lambda) V S_{0}^{-1}\right)^{m} \chi_{0}(\lambda)\left(B^{+}\right)^{\prime}(\lambda) V S_{0}^{-1}\left(\left(\chi_{1} B^{+}\right)(\lambda) V S_{0}^{-1}\right)^{n-(m+1)}
$$

Multiplication of operator-valued functions results in a convolution of their respective Fourier transforms, just as it does in the scalar case. Similarly, the $L^{1}$ theory of convolution applies in this setting provided the domain of each operator is identified with the range of its predecessor. Then

$$
\begin{aligned}
& \left\|\left[\chi_{0}\left(\tilde{B}^{+}\right)^{\prime}\right]^{\vee}\right\|_{L^{1}\left(H S\left(-1^{-},-2^{-}\right)\right)} \\
& \quad \leq \sum_{n=1}^{\infty} \sum_{m=0}^{n-1}\left\|\left(\chi_{1} B^{+}\right)^{\vee} V S_{0}^{-1}\right\|^{m}\left\|\left[\chi_{0}\left(B^{+}\right)^{\prime}\right]^{\vee} V S_{0}^{-1}\right\|\left\|\left(\chi_{1} B^{+}\right)^{\vee} V S_{0}^{-1}\right\|^{n-(m+1)}
\end{aligned}
$$

where the norms are taken in $L^{1}$ with values in $H S\left(-2^{-},-2^{-}\right), H S\left(-1^{-},-2^{-}\right)$, and $H S\left(-1^{-},-1^{-}\right)$, respectively.

From equation (40) we see that the sum converges exponentially provided $\lambda_{0}$ is chosen small enough.

Remarks. Throughout the discussion, operators have been estimated by the Hilbert-Schmidt norm as a matter of computational convenience. More precisely, we needed to know that various kernels $K(x, y)$ of $L^{2}$-bounded operators have the property that $|K(x, y)|$ again gives rise to an $L^{2}$-bounded operator (on this level of generality we do not need to distinguish between $L^{2, \sigma}$ and $L^{2}$, since the weights can be included in the kernel). Note that this property is automatic if $K(x, y)$ is HilbertSchmidt.

More generally, note that an operator of the form $I+T$ with kernel $\delta(x-y)+K(x, y)$ where $K$ is Hilbert-Schmidt, still has the property that the absolute value of the kernel gives rise to an $L^{2}$-bounded operator. Moreover, if $T$ is Hilbert-Schmidt and $(I+T)^{-1}$ exists, then $(I+T)^{-1}-I=-(I+T)^{-1} T$ is again Hilbert-Schmidt. This observation implies, in particular, that $\left|S_{0}^{-1}\right|$ is $L^{2, \sigma}$-bounded with $-\frac{5}{2}<\sigma<-\frac{1}{2}$. Here and in what follows, $|T|$ stands for the operator that is given by the absolute value of the kernel of $T$.

Consider the case when the derivative $\frac{d}{d \lambda}$ falls on a different term in (36), for example on $e^{i \lambda\left|\mathbf{y}-x_{4}\right|}$. In the lines which follow, one is then led to control the size of

$$
\sup _{\mathbf{x}, \mathbf{y} \in \mathbb{R}^{3}} \frac{1}{t^{3 / 2}}\|V\|_{L^{2,1+}}\left\|\int\left|S_{0}^{-1}\left[\chi_{0}\left(\tilde{B}^{+}\right)\right]^{\vee}(u)\right| d u\right\|_{\mathcal{B}\left(-1^{-},-1^{-}\right)}\left\||\mathbf{x}-\cdot|^{-1}\right\|_{L^{2,-1}}
$$

which depends eventually on the finiteness of the central integral

$$
\int_{-\infty}^{\infty}\left\|\left|\left[\chi_{0} \tilde{B}^{+}\right]^{\vee}(u)\right|\right\|_{\mathcal{B}\left(-1^{-},-1^{-}\right)} d u .
$$

Here $\mathcal{B}\left(-1^{-},-1^{-}\right)$stands for the bounded operators $L^{2,-1-} \rightarrow L^{2,-1-}$.

Unlike in (38), the Neumann series for $\chi_{0} \tilde{B}^{+}$begins with a zero-order term, namely $\chi_{0}(\lambda)$ times the identity map. While the identity is a bounded operator on $L^{2,-1^{-}}$it does not belong to the HilbertSchmidt class. All higher order terms are Hilbert-Schmidt, however, because they each contain at least one multiple of $B^{+}(\lambda) V S_{0}^{-1}$. A similar zero-order term appears if the derivative in (36) falls on $e^{i \lambda\left|\mathbf{x}-x_{1}\right|}$ or on the cut-off function $\chi_{0}(\lambda)$.

Acknowledgement: The second author was supported by the NSF grant DMS-0070538 and a Sloan fellowship. 


\section{References}

[Ag] Agmon, S. Spectral properties of Schrödinger operators and scattering theory. Ann. Scuola Norm. Sup. Pisa Cl. Sci. (4) 2 (1975), no. 2, 151-218.

[ArtYaj] Artbazar, G., Yajima, K. The $L^{p}$-continuity of wave operators for one dimensional Schrödinger operators. J. Math. Sci. Univ. Tokyo 7 (2000), no. 2, 221-240.

[DeiTru] Deift, P., Trubowitz, E. Inverse scattering on the line. Comm. Pure Appl. Math. XXXII (1979), 121-251.

[Jen1] Jensen, A. Spectral properties of Schrödinger operators and time-decay of the wave functions results in $L^{2}\left(R^{m}\right), m \geq 5$. Duke Math. J. 47 (1980), no. 1, 57-80.

[Jen2] Jensen, A. Spectral properties of Schrödinger operators and time-decay of the wave functions. Results in $L^{2}\left(R^{4}\right)$. J. Math. Anal. Appl. 101 (1984), no. 2, 397-422.

[JenKat] Jensen, A., Kato, T. Spectral properties of Schrödinger operators and time-decay of the wave functions. Duke Math. J. 46 (1979), no. 3, 583-611.

[JenNen] Jensen, A., Nenciu, G. A unified approach to resolvent expansions at thresholds. Rev. Math. Phys. 13 (2001), no. 6, 717-754.

[JSS] Journé, J.-L., Soffer, A., Sogge, C. D. Decay estimates for Schrödinger operators. Comm. Pure Appl. Math. 44 (1991), no. 5, 573-604.

[Kato] Kato, T. Wave operators and similarity for some non-selfadjoint operators. Math. Ann. 162 (1965/1966), 258-279.

[Katz] Katznelson, Y. An introduction to harmonic analysis. Dover, 1968.

[Rau] Rauch, J. Local decay of scattering solutions to Schrödinger's equation. Comm. Math. Phys. 61 (1978), no. 2, 149-168.

[ReedSim] Reed, M., Simon, B. Methods of modern mathematical physics. IV. Analysis of operators. Academic Press [Harcourt Brace Jovanovich, Publishers], New York-London, 1978.

[RodSch] Rodnianski, I., Schlag, W. Time decay for solutions of Schrödinger equations with rough and time-dependent potentials. to appear in Invent. Math.

[Wed1] Weder, R. $L^{p}-L^{\dot{p}}$ estimates for the Schrödinger equation on the line and inverse scattering for the nonlinear Schrödinger equation with a potential. J. Funct. Anal. 170 (2000), no. 1, 37-68.

[Wed2] Weder, R. The $W_{k, p}$-continuity of the Schrödinger wave operators on the line. Comm. Math. Phys. 208 (1999), no. 2, 507-520.

[Yaj1] Yajima, K. The $W^{k, p}$-continuity of wave operators for Schrödinger operators. J. Math. Soc. Japan 47 (1995), no. 3, 551-581.

[Yaj2] Yajima, K. L $L^{p}$-boundedness of wave operators for two-dimensional Schrödinger operators. Comm. Math. Phys. 208 (1999), no. 1, 125-152. 
Division of Astronomy, Mathematics, and Physics, 253-37 Caltech, Pasadena, CA 91125, U.S.A.

email: mikeg@caltech.edu, schlag@caltech.edu 